\title{
Electronic transport in single-helical protein molecules: Effects of multiple charge conduction pathways and helical symmetry
}

\author{
Sourav Kundu ${ }^{*}$ and S. N. Karmakar \\ Condensed Matter Physics Division, Saha Institute of Nuclear Physics, 1/AF, Bidhannagar, Kolkata 700 064, India
}

\begin{abstract}
We propose a tight-binding model to investigate electronic transport properties of single helical protein molecules incorporating both the helical symmetry and the possibility of multiple charge transfer pathways. Our study reveals that due to existence of both the multiple charge transfer pathways and helical symmetry, the transport properties are quite rigid under influence of environmental fluctuations which indicates that these biomolecules can serve as better alternatives in nanoelectronic devices than its other biological counterparts e.g., single-stranded DNA.

PACS numbers: 72.15.Rn, 73.23.-b, 73.63.-b, 87.14.gk
\end{abstract}

\section{INTRODUCTION}

Current days biomolecules are receiving huge attention from different scientific communities including physics, chemistry and others because of their possible applications in nanoelctronics and the need of understanding electronic (spin) transfer process in biological systems [14]. DNA is one of them which has attracted major attention from the beginning of the last decade [5 -9]. Whereas other biomolecules such as proteins are less attended in this respect. However with the recent progress in chiralinduced spin selectivity (CISS) both DNA and protein are getting similar attraction [10 22] across various disciplines as they both have helical structures which can be used for efficient spin polarization. In 2011 Göhler et al. [10] showed that double-stranded DNA (ds-DNA) can be used as a good spin filtering agent with length dependent spin polarization up to $60 \%$. Whereas no spin polarization was achieved for single-stranded DNA (ssDNA). These findings are then theoretically supported by Guo et al. [12]. But recent experiments suggest that $\alpha$-helical proteins are also quite efficient in spin polarization process though it has single helical structure [21, 22]. These results open up an opportunity to examine these single-helical structures from a new aspect, different models are also proposed to explain these experimental results [14]. In respect of electronic transport properties DNA is widely studied, though there are still controversies over different experimental results [9, 23 26]. Questions are still remain on reproducibility of the experimental data and underlying charge transfer mechanism [27-33]. Whereas the same properties of different protein molecules are less examined. There are few number of reports available in literature on the electronic transfer process in proteins [34 38] but no such report on the effects of environment on its electronic transport properties. It is confirmed by CISS study and related theoretical work that there are multiple charge conduction pathways (MCCP) present in single-helical pro-

*Electronic address: sourav.kunduphy@gmail.com teins due to which they are able to polarize the electron spin [14, 21, 22]. This possibility of MCCP make helical protein molecules very good agents for long-range charge transport. As proteins have these MCCP, electrons will face less disturbances/environmental effects during conduction and transport characteristics will be much rigid; reproducing experimental results will be much simpler with them.

In this paper we make an attempt to study the electronic transport properties of single helical proteins incorporating both the helical symmetry and possibility of MCCP within tight-binding framework. We propose a model Hamiltonian to explore electronic transport through single-helical proteins and compare our results with another model proposed in Ref. 14]. We study different transport properties from localization behaviour to $\mathrm{I}-\mathrm{V}$ response including the effects of environment that are modeled in terms of disordered on-site potential of the amino acids within the tight-binding Hamiltonian. Our investigations show that due to presence of MCCP the effects of environment are much smaller which enable long range coherent charge transfer in these biomolecules. Interplay of helical symmetry and disorder also has nontrivial effects on localization and $\mathrm{I}-\mathrm{V}$ responses of the protein molecules.

\section{THEORETICAL FORMULATION}

The two-terminal electronic transport through singlehelical protein molecule can be simulated using the following tight-binding Hamiltonian for the entire system

$$
H_{\text {tot }}=H_{\text {pro }}+H_{\text {leads }}+H_{\text {tun }}
$$

where $H_{\text {pro }}$ is the Hamiltonian for the protein molecule, $H_{\text {leads }}$ represents the one dimensional semi-infinite leads on the both sides of the protein molecule and $H_{\text {tun }}$ is the tunneling Hamiltonian between protein molecule and the leads. The Hamiltonian (Fig 1) for the protein molecule 
is formed on the basis set spanned by the amino acids

$$
H_{\text {pro }}=\sum_{i=1}^{N} \epsilon c_{i}^{\dagger} c_{i}+\sum_{i=1}^{N-1} t c_{i}^{\dagger} c_{i+1}+\sum_{i=1}^{N-n} t^{\prime} c_{i}^{\dagger} c_{i+n}+\text { H.c(.2.) }
$$

where $c_{i}^{\dagger}\left(c_{i}\right)$ is the creation (annihilation) operator for electrons at the $i$ th Wannier state of the protein molecule with length $\mathrm{N}, t=$ nearest neighbour hopping amplitude, $\epsilon=$ on-site potential energy of the amino acids, $t^{\prime}=$ hopping integral between two neighboring atomic sites in adjacent pitches which incorporates the possibility of MCCP along the helix. Here $n$ is the number of amino acids within a given pitch, parameter that accounts for the helical symmetry. A dispersion relation for an infinite homogeneous chain of protein molecule can be obtained following the above Hamiltonian: $E=$ $\epsilon+2 t \cos (k)+2 t^{\prime} \cos (n k)$ which explicitly depends on helical symmetry $(n)$.

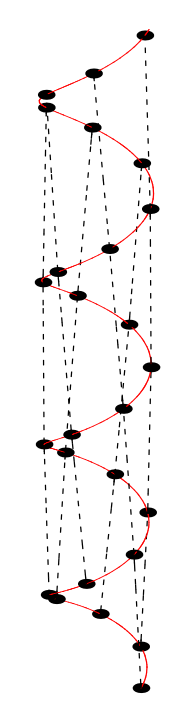

FIG. 1: (Color online). Schematic diagram of a single-helical protein molecule. Black dots on the helix represent the amino acids and dotted (black) lines between those black dots represent hopping $\left(t^{\prime}\right)$ between neighbouring amino acids of adjacent pitches. The red line shows the single helix.

Apart from the above model, we also use another model following Ref. [14] to compare our results. The protein molecule is described in this model following the tight-binding Hamiltonian (later on we refer this as model:2 throughout the paper)

$$
H_{\text {pro }}=\sum_{i=1}^{N} \epsilon c_{i}^{\dagger} c_{i}+\sum_{i=1}^{N-1} \sum_{j=1}^{N-i} t_{j} c_{i}^{\dagger} c_{i+j}+\text { H.c. }
$$

where $c_{i}^{\dagger}, c_{i}, \epsilon$ and $\mathrm{N}$ have their usual meanings. $t_{j}=$ $t_{1} e^{\left(l_{j}-l_{1}\right) / l_{c}}$ is the jth neighbouring hopping amplitude, where $l_{j}$ is the distance between to neighbour $i$ and $i+j$, $l_{c}$ is the decay exponent and $t_{1}$ is the nearest neighbour hopping integral. Here we have assumed that wave functions related to electrons decay exponentially over distance. These assumptions are similar to the SlaterKoster scheme, and $l_{c}$ can be obtained by matching to first-principle calculations [1, 14].

In order to study the transport behavior of protein molecules, we use semi-infinite $1 \mathrm{D}$ chains as leads connected to the left $(\mathrm{L})$ and right $(\mathrm{R})$ ends of the protein molecule and the corresponding Hamiltonian can be expressed as

$$
H_{\text {leads }}=\sum_{i}\left(\epsilon c_{i}^{\dagger} c_{i}+t c_{i}^{\dagger} c_{i+1}+\text { H.c. }\right) \text {, }
$$

where $i<0$ and $i>N$ respectively represent left and right semi-infinite $1 \mathrm{D}$ leads . The tunneling Hamiltonian between the leads and protein molecule is given by $H_{\text {tun }}=\tau\left(c_{0}^{\dagger} c_{1}+c_{N}^{\dagger} c_{N+1}+\right.$ H.c. $) \quad$ where $\tau$ is the tunneling matrix element between protein and the leads. In order to obtain transmission probability $T(E)$ of electron through single-helical protein we use the Green's function formalism [39]. The single particle retarded Green's function for the entire system at an energy $E$ is given by $G^{r}=(E-H+i \eta)^{-1}$, where $\eta \rightarrow 0^{+}$. The transmission probability of an electron with incident energy $E$ is given by $T(E)=\operatorname{Tr}\left[\Gamma_{L} G^{r} \Gamma_{R} G^{a}\right]$ where $\operatorname{Tr}$ represents trace over reduced Hilbert space spanned by the protein molecule. The retarded and the advanced Green's functions in the reduced Hilbert space can be expressed as $G^{r}=\left[G^{a}\right]^{\dagger}=\left[E-H_{\text {pro }}-\Sigma_{L}^{r}-\Sigma_{R}^{r}+i \eta\right]^{-1}$, where $\Sigma_{L(R)}^{r(a)}=H_{\text {tun }}^{\dagger} G_{L(R)}^{r(a)} H_{\text {tun }}$ is retarded (advanced) self energy of the left (right) lead and $\Gamma_{L(R)}=i\left[\Sigma_{L(R)}^{r}-\Sigma_{L(R)}^{a}\right]$ is the level broadening due to coupling of the leads with the protein molecule. $G_{L(R)}^{r(a)}$ being the retarded (advanced) Green's function for the left (right) lead. It can easily be shown that $\Gamma_{L(R)}=-2 \operatorname{Im}\left(\Sigma_{L(R)}^{r}\right)$, where $\operatorname{Im}$ represents the Imaginary part. At absolute zero temperature, using the Landauer formula, current through the protein molecule for an applied bias voltage $V$ is given by $I(V)=\frac{2 e}{h} \int_{E_{F}-e V / 2}^{E_{F}+e V / 2} T(E) d E$, where $E_{F}$ is the Fermi energy. We have assumed that voltage drop occurs only at the boundaries of the conductor.

\section{RESULTS:}

To perform numerical study we use following parameter values for our proposed model throughout the entire work: $\epsilon=0 \mathrm{eV}, t=1.0 \mathrm{eV}$ and $t^{\prime}=t / 10=0.1 \mathrm{eV}$. We compare our results with model: 2 using the following parameters: $l_{1}=4.1, l_{2}=5.8, l_{3}=5.1, l_{4}=6.2, l_{5}=8.9, l_{6}=10.0$ and $l_{c}$ is taken as 0.9 , all units are in $\AA$. Using these values we can calculate the related hopping integrals $\left(t_{j}\right)$ which gives $t_{2} \sim 0.16 t_{1}$ and so on. It is clear that gradually $t_{j}$ values will decrease (except $t_{3}>t_{2}$ ) with in- 
creasing distance, therefore we restrict ourselves to $t_{6}$ and set $t_{1}=t=1.0 \mathrm{eV}$. These parameter values for model: 2 are extracted from Ref. 14]. For ss-DNA, to calculate its transport properties, we set $t^{\prime}=0 \mathrm{eV}$ in our model which cancels any possibility of MCCP. We first study the localization properties of the system. The localization length $(l)$ of the system is calculated from the Lyapunov exponent $(\gamma)$ [40]

$$
\gamma=1 / l=-\lim _{N \rightarrow \infty} \frac{1}{2 N}<\ln (T(E))>,
$$

where $N=$ length of the system and $<>$ denotes average over different disorder configurations. In actual experiments there are various environmental fluctuations that we have simulated in the model by considering the on-site energy $\epsilon$ to be randomly distributed within the range $[\epsilon-\mathrm{w} / 2, \epsilon+\mathrm{w} / 2]$, where $\mathrm{w}$ represents the disorder strength.

In fig. 2 we show the variation of Lyapunov exponent with disorder at different energies for both the protein molecule and ss-DNA. For $n=3$, at low disorder there is almost no variation in the localization length for different energies. With increasing disorder the separation between different energies become distinguishable, energies close to the band centre become more localized than the band edges. For $n=5$, variation between different energies are maximum at low disorder which gradually vanishes with increasing disorder. For model:2, different energies show different localization length for almost entire range of disorder with a crossover point around $\mathrm{w}=5$. At these disorder strength, localization length corresponding to different energies become nearly equal. For ss-DNA, different energies remain separated throughout the entire range and the separation between them decreases slightly at higher disorder. It is clear form these plots that $n=5$ has the maximum localization length for entire disorder range, which shows it is the most favourable conformation of the protein molecule for charge transport.

Fig. 3 presents variation of $\gamma$ with energy at different disorder strength for different models and molecules. For protein molecule with $n=3$, at low disorder system is less localized and $\gamma$ is close to zero. With increasing disorder, $\gamma$ increases and the system becomes more and more localized. However, at energies close to band centre $(\mathrm{E}=0$ $\mathrm{eV}$ ) localization is stronger than that at the band edges which is also evident from previous plots (Fig. 2). For $n=5$, there is a typical shape in $\gamma$ within the energy region 1 to $1.5 \mathrm{eV}$ (both -ve and +ve) which shows that at these energies system is maximally localized even at low disorder, with increasing disorder these features die out gradually. These specific features are results of their helical structure, the dispersion (E-k) of the molecule explicitly depends on the helical symmetry $(n)$ (so the density of states also) and with changing $n$ specific features appear in different transport parameters. For model:2, there exists also the same feature at low disorder but near the band centre which vanishes with increasing dis-
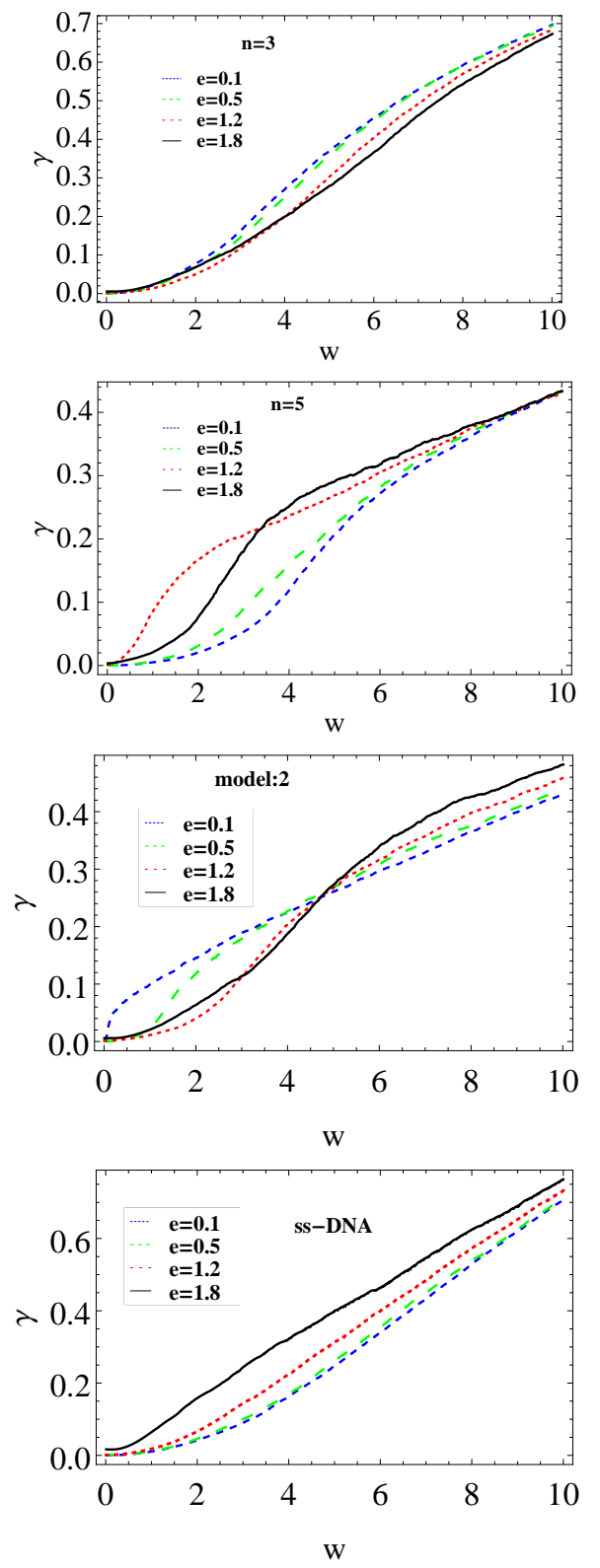

FIG. 2: (Color online). Lyapunov exponent $(\gamma)$ vs disorder (w) for $n=3$ and $n=5$, model:2 and for ss-DNA at different energies (e). Figure shows that $n=5$ is maximally extended with lowest $\gamma$ value for all the energies.

order. This feature suggest that there is deficiency of accessible states around the band centre, with increasing disorder new states generates in these region at the cost of the other states around band edges and this feature vanishes. Another point to be noted that $\gamma$ is smaller in -ve energy region than +ve region, which indicates that the band is not symmetric with respect to energy and therefore effect of disorder is different on both side of $\mathrm{E}=0 \mathrm{eV}$. For ss-DNA, $\gamma$ increases with disorder, band edges are more localized than the band centre which is trivial in this case.

In fig. 4 we show the I-V characteristics of the pro- 

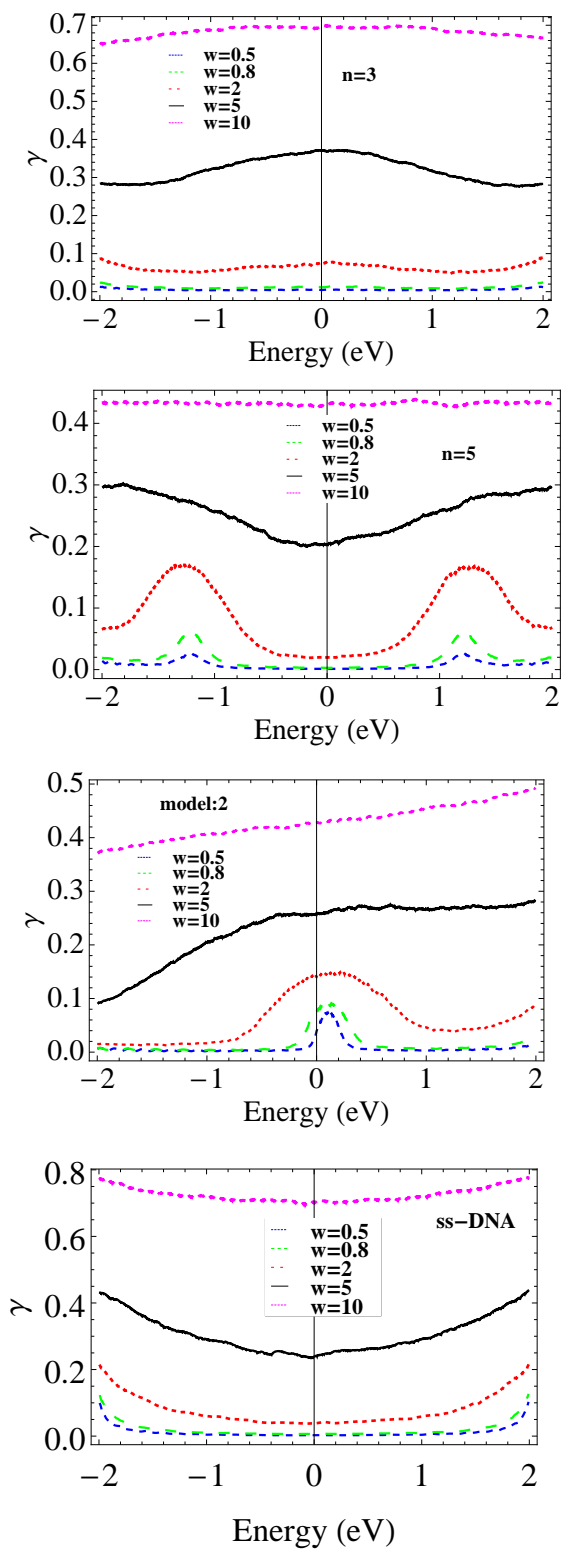

FIG. 3: (Color online). Lyapunov exponent $(\gamma)$ vs Energy for $n=3$ and $n=5$, model:2 and for ss-DNA at different disorder strength. Some specific features of the curve can be seen for $n=5$, and model:2. It is evident that ss-DNA being the most localized medium throughout the entire energy range.

tein molecule using our proposed model for $n=3$ and 5 . It is clear from the plots that for low disorder, current is almost linear and with increasing disorder $(\mathrm{w}=5)$ system becomes insulating. One difference is to be noted between $n=3$ and 5 , current output is always greater for $n=5$ than $n=3$. This difference becomes more distinguishable under environmental fluctuations. If we increase $n$, electrons get more number of pathways to hop from one site to another. In other words, increasing $n$ provides a shortcut pathway for electrons to move along the helix from one pitch to the next bypassing all other amino acids in that pitch. Due to this galloping elec-
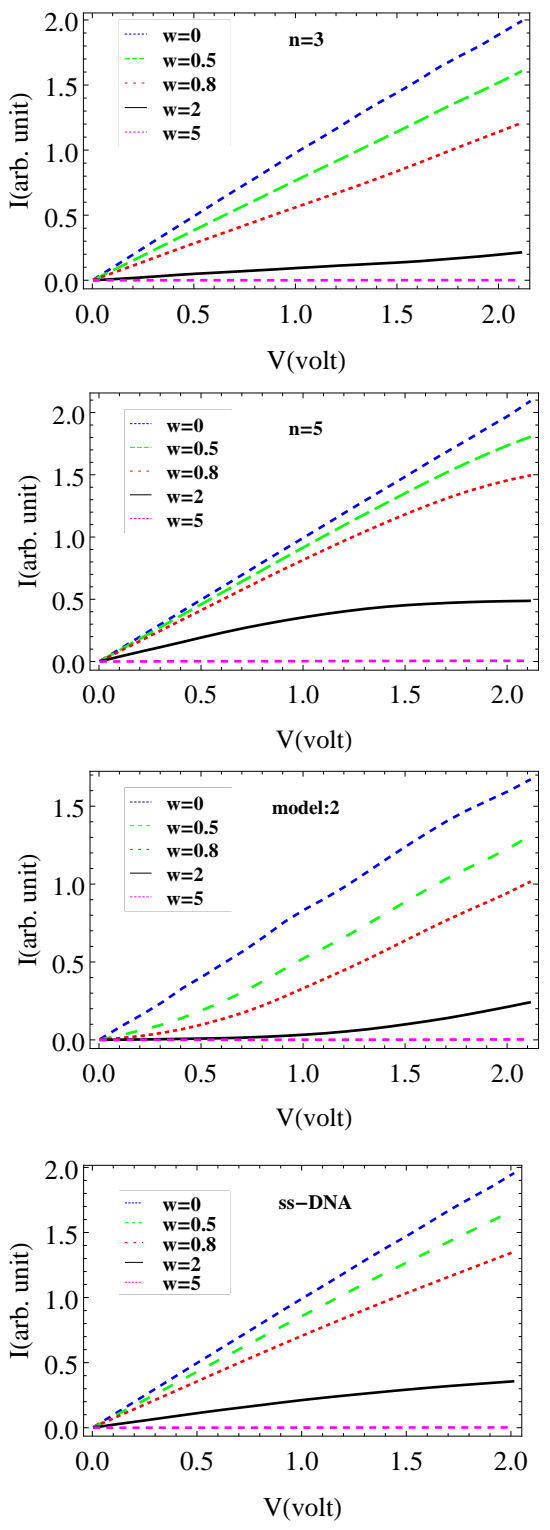

FIG. 4: (Color online). I-V characteristics for different models and molecules. For $\mathrm{w}=0$, all of the curves are nearly similar, with increasing disorder the responses become differentiable. It is clear from the plots that $n=5$ gives maximum current output under environmental fluctuations, that again shows it is the best possible conformation for the protein molecule for charge transport.

trons encounter less scattering, hence the current increases. For $n=5$ at $\mathrm{w}=2, \mathrm{I}-\mathrm{V}$ response is linear at low bias but it saturates with increasing bias voltage which signifies that there are no more accessible states available to conduct electrons at higher bias. For model:2, at low disorder there is not so much variation in the current response with our model, but for appreciable disorder $(\mathrm{w}=2)$ response become semiconducting with cut-off voltage around 0.5 Volt, in contrast with linear response achieved in our model. This variation of current is due 
to the difference in these two models. The model:2 only caters the possibility of multiple charge conduction pathways but neglect the helical symmetry which is a fundamental structure of these biomolecules. Our model also incorporates this symmetry, due to which electron transport enhances and current output is always greater in our case $(n=5)$. The $\mathrm{I}-\mathrm{V}$ responses of the protein molecule under environmental effects match qualitatively well with experimental findings [34, 41, 42]. For ss-DNA current output is quite similar with the protein molecule for clean case $(\mathrm{w}=0)$, but as soon as we apply disorder current decreases rapidly. If we compare ss-DNA with protein molecule $(n=5)$, one can easily see that even under small disorder $(\mathrm{w}=0.5,0.8)$ there is substantial drop in the current output for ss-DNA. This shows that single-helical proteins are much better conductor under external disturbances than ss-DNA.

\section{CONCLUSION:}

We present a detail analysis of electronic transport properties of single-helical protein molecules based on tight-binding framework. Though with recent progress in CISS single helical proteins are getting considerable attention these days but their basic electronic transport properties remain quite under-explored. We show that due to existence of both the helical symmetry and MCCP transport properties are less affected by external distur- bances and these biomolecules can serve as a better alternative in nanoelectronics than its DNA counterparts (ssDNA) or other artificial semiconductor nanowires which do not contain the possibility of MCCP. As a consequence of these features long range coherent electron transport can be possible in these biomolecules and effect of environment will also be lesser. We also show that by varying $n$ i.e., by twist-stretching of the protein molecules one can get different responses (current outputs) under environmental effects. As an example, for $n=5$ system is more conducting than $n=3$. We compare our results with the model proposed by Guo et al. 14], that matches qualitatively well in almost every aspect. But as our model includes both MCCP and helical symmetry (both are actually present in single-helical proteins) it provides more number of conduction channels and system becomes less localized. Due to helical symmetry $(n)$ the behaviour of localization properties also change which shows that using twist-stretching or conformal changes one can use a protein molecule in different ways. We check our results for other model parameters also e.g., $l_{c}=0.4$ and 1.4 $\AA$ 14] but the qualitative behaviour remains the same. In summary our study shows the rigidity of transport properties of the single-helical protein molecules even under environmental effects due to presence of both the helical symmetry and MCCP, which promises that they can be used in future nanoelectronic devices with much more reliability.
[1] R. G. Endres, D. L. Cox and R. R. P. Singh, Rev. Mod. Phys. 76, 195 (2004).

[2] I. utic, J. Fabian and S. D. Sarma, Rev. Mod. Phys. 76, 323 (2004).

[3] J. C. Genereux and J. K. Barton, Chem. Rev. 2010, 110, 1642 (2010).

[4] M. Cordes and B. Giese, Chem. Soc. Rev. 38, 892 (2009).

[5] S. O. Kelley and J. K. Barton, Science 283, 375 (1999).

[6] H. W. Fink and C. Schönenberger, Nature (London) 398, 407 (1999).

[7] D. Porath, A. Bezryadin, S. De Vries and C. Decker, Nature (London) 403, 635 (2000).

[8] L. Cai, H. Tabata and T. Kawai, Appl. Phys. Lett. 77, 3105 (2000).

[9] P. Tran, B. Alavi and G. Grüner, Phys. Rev. Lett. 85, 1564 (2000).

[10] B. Göhler et al., Science 331, 894 (2011).

[11] Z. Xie et al., Nano Lett. 11, 4652 (2011).

[12] A-M Guo and Q-F Sun, Phys. Rev. Lett. 108, 218102 (2012).

[13] A-M Guo and Q-F Sun, Phys. Rev. B 86, 035424 (2012).

[14] A-M Guo and Q-F Sun, Proc. Natl. Acad. Sci. USA 111, 11658 (2014).

[15] D. Rai and M. Galperin, J. Phys. Chem. C 117, 13730 (2013).

[16] A. A. Eremko and V. M. Loktev Phys. Rev. B 88, 165409 (2013).

[17] S. Yeganeh, M. A. Ratner, E. Medina and V. Mujica, J.
Chem. Phys. 131, 014707 (2009).

[18] R. Gutierrez, E. Díaz, R. Naaman and G. Cuniberti, Phys. Rev. B 85, 081404 (2012).

[19] K. Senthilkumar, N. Kantor-Uriel, S. P. Mathew, R. Guliamov and R. Naaman, Phys. Chem. Chem. Phys. 15, 18357 (2013).

[20] J. Gersten, K. Kaasbjerg and A. Nitzan, J. Chem. Phys. 139, 114111 (2013).

[21] D. Mishra et al., Proc. Natl. Acad. Sci. USA 110, 14872 (2013).

[22] O. Ben Dor, S. Yochelis, S. P. Mathew, R. Naaman and Y. Paltiel, Nat. Commun. 4, 2256 (2013).

[23] Y. Zhang, R. H. Austin, J. Kraeft, E. C. Cox and N. P. Ong, Phys. Rev. Lett. 89, 198102 (2002).

[24] A. J. Storm et al., Appl. Phys. Lett. 79, 3881 (2001).

[25] K. H. Yoo et al., Phys. Rev.Lett. 87, 198102 (2001).

[26] A. Y. Kasumov et al., Science 291, 280 (2001).

[27] E. M. Conwell and S. V. Rakhmanova, Proc. Natl. Acad. Sci. USA 97, 4557 (2000).

[28] C. Dekker and M. A. Ratner, Physics World 14(8): 29-33 (2001).

[29] M. A. Ratner, Nature (London) 397, 480 (1999).

[30] D. N. Beratan, S. Priyadarshy and S. M Risser, Chem. Biol. 4, 3 (1997).

[31] S. Kundu and S. N. Karmakar, Phys. Rev. E 89, 032719 (2014).

[32] S. Kundu and S. N. Karmakar, Phys. Lett. A 379, 1377 (2015). 
[33] S. Kundu and S. N. Karmakar, AIP Advances 5, 107122 (2015).

[34] Y. Jin, N. Friedman, M. Sheves, T. He and D. Cahen, Proc. Natl. Acad. Sci. USA 103, 8601 (2006).

[35] T. R. Prytkova, I. V. Kurnikov and D. N. Beratan, Science 315, 5812 (2007).

[36] D. N. Beratan and I. V. Balabin, Proc. Natl. Acad. Sci. USA 105, 403 (2008).

[37] J. Gao et al, Angew. Chem. Int. Ed. Engl.50, 1926 (2011).

[38] L. Sepunaru, N. Friedman, I. Pecht, M. Sheves and D.
Cahen, J. Am. Chem. Soc. 134, 4169 (2012).

[39] S. Datta, Electronic transport in mesoscopic systems, Cambridge University Press, Cambridge (1995).

[40] M. D. Ventra, Electrical transport in nanoscale system, Cambridge University Press, Cambridge (2008).

[41] T. Lee, W. Wang, J. F. Klemic, J. J. Zhang, J. Su and M. A. Reed, J. Phys. Chem. B 108, 8742 (2004).

[42] I. Ron, L. Sepunaru, S. Itzhakov, T. Belenkova, N. Friedman, I. Pecht, M. Sheves and D. Cahen, J. Am. Chem. Soc. 132, 4131 (2010). 